\title{
Nanoazitromicina preparada por el método solvente/ antisolvente: efecto sobre la disolución
}

\author{
JONATHAN DIEGO HURTADO JARRINa* ${ }^{*}$, JAVIER RODRIGO SANTAMARÍA-AGUIRREa \\ aFacultad de Ciencias Químicas, \\ Universidad Central del Ecuador (UCE), \\ *Correspondencia: jonathanhurtado65@gmail.com
}

Recibido: 28 agosto 2017. Aceptado: 20 noviembre 2017

\section{Resumen}

La azitromicina pertenece al grupo Il del sistema de clasificación bioframaceútico, su baja solubilidad acuosa explica su pobre biodisponibilidad. El propósito del presente trabajo fue desarrollar una nanosuspensión de azitromicina por el método solvente/antisolvente para mejorar su velocidad de disolución, para ello se determinó la influencia de la temperatura del medio de dispersión. Una solución etanólica de azitromicina $(200 \mathrm{mg} / \mathrm{ml})$ se adicionó a una velocidad de $100 \mathrm{ul} / \mathrm{s}$, sobre una solución acuosa de tween $80(4,5 \mathrm{mg} / \mathrm{ml})$ a 0, 25 y $80^{\circ} \mathrm{C}$. Se obtuvieron nanopartículas de azitromicina de 268,2 nm, con un índice de polidispersión de 0,396, potencial Z de -39,6 mV y 94,2\% de pureza. Con ellas se elaboró polvo para reconstituir suspensión oral utilizando hidroxietilcelulosa como coloide protector y goma xantan como viscosante. La velocidad de disolución de las nanopartículas de azitromicina es casi 400\% mayor que la azitromicina en polvo.

Palabras clave: nanosuspensión, azitromicina, solvente / antisolvente.

\section{Nano azithromycin prepared by the solvent / antisolvent method: effect on dissolution}

\section{Abstract}

Azithromycin belongs to group II of the biopharmaceutical classification system, its low aqueous solubility explains its poor bioavailability. The purpose of the present work was to develop a Nano suspension of azithromycin by the solvent / antisolvent method to improve its dissolution speed, for this, the influence of the temperature of the dispersion means was determined. An ethanolic solution of azithromycin $(200 \mathrm{mg} / \mathrm{ml})$ was added at a rate of $100 \mathrm{ul} / \mathrm{s}$, on an aqueous solution of tween $80(4.5 \mathrm{mg} / \mathrm{ml})$ at 0,25 and $80^{\circ} \mathrm{C}$. Azithromycin nanoparticles of $268.2 \mathrm{~nm}$ were obtained, with a polydispersity index of $0.396, Z$ potential of $-39.6 \mathrm{mV}$ and $94.2 \%$ purity. With them, powder was made to reconstitute oral suspension using hydroxyethyl cellulose as a protective colloid and xanthan gum as a viscosifier. The dissolution speed of azithromycin nanoparticles is almost $400 \%$ higher than azithromycin powder.

Key words: nanosuspension, azithromycin, solvent / antisolvent. 


\section{Introducción}

Es sumamente raro encontrar fármacos que se administren como sustancias puras, por lo contrario, en su mayoría se administran en forma de preparados farmacéuticos o medicamentos, que puede variar desde soluciones relativamente sencillas a sistemas complejos conseguidos mediante el uso de aditivos y excipientes adecuados; existen un sin número de formas farmacéuticas en las cuales se puede incorporar un fármaco para tratar de manera eficiente y cómoda una enfermedad, los fármacos son diseñados para ser administrados por diferentes vías, con la finalidad de conseguir una respuesta terapéutica máxima [1].
La vía oral es la más utilizada, cerca del $40 \%$ de los medicamentos la emplean [2]; en comparación con otras vías es sencilla, más cómoda y de mayor seguridad, sin embargo, una de las limitantes más importantes al momento de diseñar formas farmacéuticas orales, se da cuando el proceso de disolución es un paso limitante para la absorción del fármaco en el organismo; efecto que se ve agravado cuando en fármacos poco solubles o insolubles, llegando incluso a no obtenerse el efecto terapéutico deseado debido a su baja biodisponibilidad; los fármacos más susceptibles son aquellos que pertenecen al grupo II del Sistema de Clasificación Biofarmacéutico (BCS): alta permeabilidad, baja solubilidad y los del grupo IV: baja permeabilidad, baja solubilidad [3].

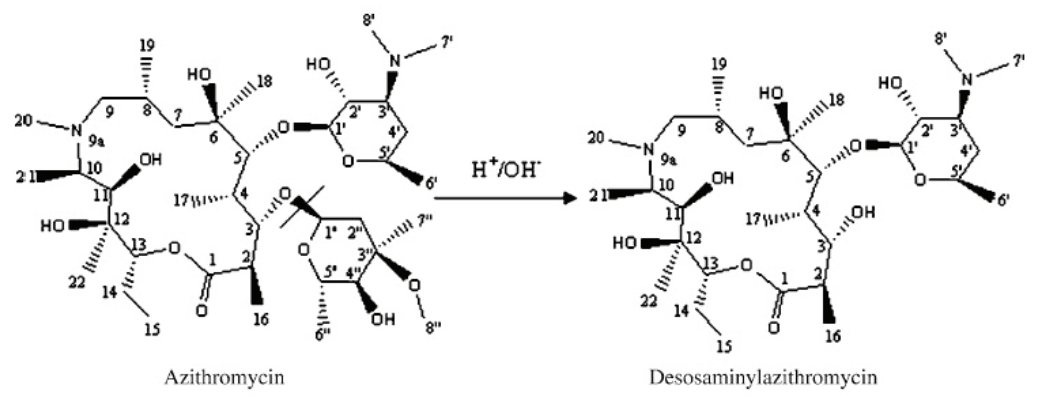

Figura 1. Estructura química y degradación de la azitromicina [5].

En la figura 1 se muestra la estructura de la azitromicina que consiste en un anillo de lactona macrocíclica de 15 miembros, al que se encuentran unidos dos azúcares, un aminoazucar, d-desosamina mediante un enlace $\beta$-glucosídico a la posición C5 del anillo de lactona y un azúcar neutro, I-cladinosa a través de un enlace $\alpha$-glucosídico a la posición C3 de la lactona [5].

Tras la administración por vía oral a voluntarios sanos, la biodisponibilidad es aproximadamente del 37\% y la unión a proteínas oscila alrededor del 50\% [6].

La Azitromicina se degrada en medio acuoso, en la figura 1 también se observa el principal producto de degradación, desosaminilazitromicina que se forma por la división del enlace $\alpha$-glucosídico entre el azúcar l-cladinosa y la lactona [5].

Una solución tradicional para este problema ha sido formular comprimidos o suspensiones, estas últimas son dispersiones gruesas en las que partículas insolubles del principio activo, generalmente mayores a 1 $\mu \mathrm{m}$, se encuentran distribuidas en un medio líquido; las suspensiones no están exentas de limitantes: pobre o casi nula solubilidad, baja velocidad de disolución, inestabilidad física, reducida área superficial y gran tamaño de partícula; teniendo en cuenta que alrededor del $40 \%$ de los nuevos fármacos generados en los programas de investigación son lipófilos o poco solubles en agua [7], su incorporación en este tipo de sistema de entrega supone un reto importante para la industria farmacéutica.

En las últimas décadas la ingeniería de nanopartículas ha tomado alta relevancia en aplicaciones farmacéuticas [8], intentando resolver los problemas asociados con las limitantes descritas anteriormente; la nanotecnología permite obtener partículas de $10^{-9} \mathrm{~m}$ mediante el proceso de abajo-arriba o de arriba-abajo; al reducir el tamaño de las partículas se incrementa la superficie específica y por ende su velocidad de disolución, absorción en el organismo, la uniformidad del contenido y estabilidad física en sistemas heterogéneos.

El proceso de abajo-arriba implica una técnica de precipitación solvente/antisolvente, en la que el fár- 
maco se disuelve primero en un disolvente orgánico y después se precipita en presencia de un estabilizante. El uso de equipos simples y de bajo costo es la principal ventaja de la técnica de precipitación. En este proceso, se requiere la adición de tensoactivo para evitar la coagulación de nanopartículas en micropartículas. El fármaco necesita ser soluble en al menos un disolvente, que debe ser miscible con otro no disolvente. Sin embargo, esta técnica no es aplicable a fármacos que son poco solubles tanto en medios acuosos como no acuosos [9].

Por esta razón el uso de esta nueva tecnología en la producción nacional de medicamentos propone un enfoque viable para formular fármacos poco solubles, con un aumento de la biodisponibilidad que podría conducir a una disminución de la dosis, así como de los costos, incrementando la accesibilidad de los medicamentos a las poblaciones de escasos recursos.

\section{Parte experimental}

En una primera etapa se investigó cómo influye la temperatura del medio de dispersión en la obtención de nanopartículas de azitromicina por el método solvente/antisolvente; para lo cual se ensayó la temperatura del medio de dispersión a 0,25 y $80^{\circ} \mathrm{C}$.

Para determinar la influencia del tamaño de partícula de azitromicina sobre la velocidad de disolución, se elaboraron polvos para reconstituir suspensiones orales variando el tamaño de partícula entre azitromicina y nanoazitromicina, se caracterizó el volumen de sedimentación, contenido, potencial Z y se determinó su perfil de disolución.

\section{Procedimiento:}

\section{Elaboración de nanopartículas por el método solvente/antisolvente}

Se preparó una solución madre de azitromicina $(200$ $\mathrm{mg} / \mathrm{ml}$ ) en etanol al $96 \%$, y se filtró a través de una membrana de PVDF de 0,45 $\mu$ m; esta solución se adicionó con una jeringuilla de $3 \mathrm{ml}$ con aguja \# 21, a una velocidad de $100 \mu \mathrm{l} / \mathrm{s}$ sobre una solución de Tween $80(4,5 \mathrm{mg} / \mathrm{ml})$ agitada a $3200 \mathrm{rpm}$; se mantuvo la agitación durante 5 minutos y el secado se realizó a $30^{\circ} \mathrm{C}$ durante 48 horas en lecho estático.

\section{Elaboración de la nanosuspensión}

Se preparó el polvo para reconstituir suspensiones orales utilizando: azitromicina dihidrato, sacarosa, sodio fosfato tribásico anhidro, hidroxietilcelulosa (NATROSOL 250HX Pharm), goma xantan y los aromas cereza, vainilla y plátano (MAGIC FLAVORS); tanto los excipientes como el activo fueron tamizados en malla $0,85 \mathrm{~mm}$ y mezclados.

\section{Resultados y discusión}

\section{Efecto de la temperatura del medio de dis- persión}

Las nanopartículas secas fueron resuspensidas con una concentración de $24,5 \mathrm{mg} / \mathrm{ml}$ en agua tipo 1 y llevadas al ultrasonido por 5 minutos.

En la tabla 1 se resumen las variables respuesta para cada condición de temperatura, cada valor representa el promedio de las 3 réplicas de cada tratamiento.

Tabla 1. Resumen de la caracterización de las nanopartículas de azitromicina obtenidas a tres diferentes temperaturas.

\begin{tabular}{cccc}
\hline Temperatura, ${ }^{\circ} \mathbf{C}$ & $\begin{array}{c}\text { Tamaño de } \\
\text { partícula, } \mathbf{n m}\end{array}$ & $\begin{array}{c}\text { Índice de } \\
\text { polidispersión }\end{array}$ & Potencial Z, mV \\
\hline 0 & 100,2 & 0,484 & $-31,8$ \\
25 & 157,1 & 0,523 & $-45,1$ \\
80 & 1953,3 & 3,324 & $-15,5$ \\
\hline
\end{tabular}

Se obtuvieron nanopartículas cuando el medio de dispersión se encontraba a 0 y $25^{\circ} \mathrm{C}$; sus índices de polidispersión fueron semejantes y los potenciales $Z$ inferiores a $-30 \mathrm{mV}$. Se seleccionó el tratamiento a $25^{\circ} \mathrm{C}$ pues al trabajar a temperatura ambiente no se necesita energía para poder disminuir la temperatura del medio de dispersión lo cual conlleva al ahorro de recursos. 
Elaboración y caracterización de las nanopartículas de azitromicina obtenidas con el mejor tratamiento

Una vez definido el tratamiento para elaborar las nanopartículas, se preparó un lote tomando en cuenta la cantidad necesaria para poder realizar todos los estudios. Los resultados de la caracterización representan el promedio de 3 mediciones la cual se describe en la Tabla 2 y la Figura 2.

Tabla 2. Caracterización de las nanopartículas de azitromicina obtenidas

\begin{tabular}{cccc}
\hline $\begin{array}{c}\text { Tamaño de } \\
\text { partícula, } \mathbf{n m}\end{array}$ & $\begin{array}{c}\text { Índice de } \\
\text { polidispersión }\end{array}$ & Potencial Z, mV & Pureza, \% \\
\hline 268,2 & 0,396 & $-39,6$ & $94,2 \%$ \\
\hline
\end{tabular}

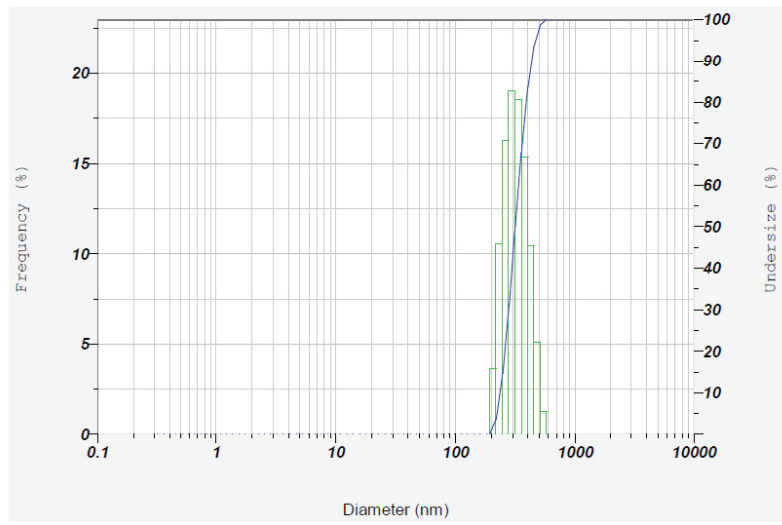

Figura 2. Tamaño de partícula de las nanopartículas determinado en DLS

Perfiles de disolución entre suspensiones de azitromicina vs nanopartículas de azitromicina
La tabla 3 detalla los resultados obtenidos en los perfiles de disolución, mismos que se grafican en la Figura 4.

Tabla 3. Porcentaje de disolución de las suspensiones preparadas con azitromicina y nanoazitromicina.

\begin{tabular}{ccc}
\hline $\begin{array}{c}\text { Tiempo } \\
\text { minutos }\end{array}$ & $\begin{array}{c}\text { Azitromicina } \\
\text { (AZT SUSP) }\end{array}$ & $\begin{array}{c}\text { Nanopartículas } \\
\text { (NAZT SUSP) }\end{array}$ \\
\hline 5 & 21,4 & 81,4 \\
10 & 32,6 & 93,3 \\
15 & 42,9 & 95,5 \\
25 & 54,7 & 97,8 \\
35 & 65,0 & 97,5 \\
\hline
\end{tabular}




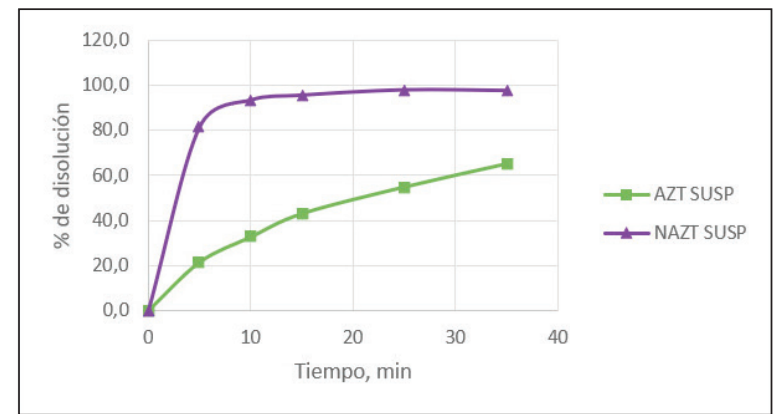

Figura 3. Perfiles de disolución de las suspensiones preparadas con azitromicina (AZT SUSP) y nanoazitromicina (NAZT SUSP).

Existe una gran diferencia entre los dos perfiles de disolución: la suspensión de nanoazitromicina liberó a los 5 minutos el 81,4\% mientras que la suspensión de azitromicina solo el 21,4\%; esta diferencia se debe no solo a la reducción del tamaño de partícula, sino también, al hecho de que las par- tículas de nanoazitromicina incluyen al tween 80 en su composición, favoreciéndose la humectación y la disolución. Según la USP 39 para los productos que se disuelven muy rápidamente $\geq 85 \%$ de disolución en 15 minutos) no es necesario el perfil de comparación.

\section{Comparación entre los parámetros caracte- rizados en la suspensión de azitromicina $y$ nanoazitromicina}

Tabla 4. Parámetros caracterizados en la suspensión de azitromicina y nanopartículas de azitromicina.

\begin{tabular}{cccc}
\hline Suspensión & Contenido, \% & Potencia Z, mV & $\begin{array}{c}\text { Volumen de } \\
\text { sedimentación }\end{array}$ \\
\hline Azitromicina & 103,8 & $-35,0$ & 0,94 \\
Nanopartículas & 104,6 & $-39,6$ & 1,00 \\
\hline
\end{tabular}

Al analizar los datos detallados en la tabla 4, podemos observar que las suspensiones de azitromicina y nanoazitromicina cumplen con las especificaciones planteadas, en contenido no mayor que $110 \%$ ni menor que $90 \%$, en potencial $Z$ valores superiores a $+30 \mathrm{mV}$ 。 inferiores a $-30 \mathrm{mV}$ y volumen de sedimentación $\geq 0,8$.

\section{Conclusiones}

Se elaboraron nanopartículas de azitromicina usando como medio de dispersión una solución acuosa de tween $80(4,5 \mathrm{mg} / \mathrm{ml})$, a 0,25 y $80^{\circ} \mathrm{C}$ y se determinó que la variable temperatura influye de una forma directamente proporcional sobre el tamaño de partícula de azitromicina; es decir a menor temperatura del medio de dispersión se pueden obtener partículas que se encuentran en escala nanométrica.

Las nanopartículas de azitromicina, obtenidas por el método solvente/antisolvente usando medio de dispersión a $25^{\circ} \mathrm{C}$, presentaron un tamaño de $268,2 \mathrm{~nm}$, índice de polidispersión 0,396, potencial Z de - 39,6 $\mathrm{mV}$ y $94,2 \%$ de pureza.

Se desarrolló un polvo para reconstituir suspensiones orales usando nanopartículas de azitromicina, con una velocidad de disolución 3,8 veces mayor que la suspensión de azitromicina original. 


\section{Referencias}

[1] Aulton, M. E. (2004). La ciencia del diseño de las formas farmacéuticas. Madrid: Elsevier.

[2] Lim Chin, W. W., \& Parmentier, J. (2014). A Brief Literature and Patent Review of Nanosuspensions to a Final Drug Product. Journal of Pharmaceutical Sciences, 2980-2999

[3] Brito, Y. (2015). Clasificación Biofarmacéutica Provisional de los Ingredientes Farmacéuticos Activos de los Sólidos Orales de Liberación Inmediata del Cuadro Básico de Medicamentos de Cuba. Santa Clara: Ediciones Alarcos.

[4] Aceituno, A. (23 de 01 de 2013). Decretos 864 y 981: productos afectos, plazos y requerimientos. Obtenido de Departamento Agencia Nacional de Medicamentos Chile: http://www.ispch.cl/sites/default/files/Decretos\%20864\%20y\%20981\%20(AAA).pdf

[5] Zhang, Y. (2009). Aspects of Degradation Kinetics of Azithromycin in Aqueous Solution. Chromatographi, 67-63.

[6] AEMPS. (01 de 2011). Agencia Española de Medicamentos y Productos Sanitarios. Obtenido de Gobierno de España: https://www.aemps. gob.es/cima/pdfs/es/ft/61272/FT_61272.pdf

[7] Patel VR, A. Y. (2011). Nanosuspension: An approach to enhanche solubility of drugs. Journal of Advanced Pharmaceutical Technology \& Research, 81-87.

[8] Sheth P, S. H. (2012). Nanoparticles in the pharmaceutical industry and the use of supercritical fluid technologies for nanoparticle production. PublMed, 269-284.

[9] Shah, D., \& Murdande, S. (2015). A Review: Pharmaceutical and Pharmacokinetic Aspect of Nanocrystalline Suspensions. ELSEVIER, 10-24. 